\title{
Are lactoferrin receptors in Gram-negative bacteria viable vaccine targets?
}

\author{
Clement Chan - Vahid F. Andisi - Dixon Ng - Nick Ostan • Warren K. Yunker • \\ Anthony B. Schryvers
}

Received: 2 January 2018/ Accepted: 28 April 2018/Published online: 16 May 2018

(C) Springer Science+Business Media, LLC, part of Springer Nature 2018

\begin{abstract}
A number of important Gram-negative pathogens that reside exclusively in the upper respiratory or genitourinary tract of their mammalian host rely on surface receptors that specifically bind host transferrin and lactoferrin as a source of iron for growth. The transferrin receptors have been targeted for vaccine development due to their critical role in acquiring iron during invasive infection and for survival on the mucosal surface. In this study, we focus on the lactoferrin receptors, determining their prevalence in pathogenic bacteria and comparing their prevalence in commensal Neisseria to other surface antigens targeted for vaccines; addressing the issue of a reservoir for vaccine escape and impact of vaccination on the microbiome. Since the selective release of the surface lipoprotein lactoferrin binding protein B by the NalP protease in Neisseria meningitidis argues against its utility as a vaccine target, we evaluated the release of outer membrane vesicles, and transferrin
\end{abstract}

C. Chan · V. F. Andisi · D. Ng · A. B. Schryvers $(\bowtie)$ Department of Microbiology, Immunology \& Infectious Diseases, University of Calgary, Calgary, AB T2N 4N1, Canada

e-mail: schryver@ucalgary.ca

N. Ostan

Department of Biochemistry, University of Toronto, Toronto, ON M5S 1A8, Canada

W. K. Yunker

Department of Surgery, University of Calgary, Calgary, AB T2N 4N1, Canada and lactoferrin binding in N. meningitidis and Moraxella catarrhalis. The results indicate that the presence of NalP reduces the binding of transferrin and lactoferrin by cells and native outer membrane vesicles, suggesting that NalP may impact all lipoprotein targets, thus this should not exclude lactoferrin binding protein $\mathrm{B}$ as a target.

Keywords Lactoferrin-binding protein - Transferrin binding protein $\cdot$ Vaccine $\cdot$ Antimicrobial peptides

\section{Introduction}

A number of Gram-negative bacterial pathogens responsible for important diseases in humans and food production animals reside exclusively in the upper respiratory or genitourinary tract of their vertebrate host and rely on surface receptors capable of specifically binding the host iron-binding glycoproteins transferrin ( $\mathrm{Tf}$ ) or lactoferrin $(\mathrm{Lf})$ to acquire iron for growth (Table 1). The typical bipartite Tf receptor is comprised of a surface anchored lipoprotein, $\mathrm{TbpB}$ (transferrin binding protein $\mathrm{B}$ ), responsible for initial capture of $\mathrm{Tf}$ and delivering it to the integral outer membrane protein TbpA (transferrin binding protein A), which extracts iron and transports it across the outer membrane (Morgenthau et al. 2013) (Fig. 1). The energy for the outer membrane transport process is derived from interaction with the TonB protein, a 
Table 1 Prevalence of transferrin and lactoferrin receptors in human and animal pathogens

\begin{tabular}{|c|c|c|c|c|c|}
\hline Host & Pathogen & Disease & $\begin{array}{l}\text { Tf } \\
\text { Receptor }\end{array}$ & $\begin{array}{l}\mathrm{Lf} \\
\text { receptor }\end{array}$ & References \\
\hline \multirow[t]{5}{*}{ Human } & Neisseria meningitidis & Meningitis, sepsis & Yes & Yes & Schryvers and Morris (1988a) \\
\hline & Neisseria gonorrhoeae & Gonorrhea & Yes & Yes & Schryvers and Lee (1989) \\
\hline & $\begin{array}{l}\text { Haemophilus influenzae (type } \\
\text { b) }\end{array}$ & $\begin{array}{l}\text { Meningitis, sepsis, } \\
\text { pnuemonia }\end{array}$ & yes & & $\begin{array}{l}\text { Schryvers and Gray-Owen } \\
\text { (1992) }\end{array}$ \\
\hline & Haemophilus influenzae (NT) & $\begin{array}{l}\text { Otitis media, COPD, } \\
\text { pneumonia }\end{array}$ & Yes & & $\begin{array}{l}\text { Gray-Owen and Schryvers } \\
\text { (1995) }\end{array}$ \\
\hline & Moraxella catarrhalis & Otitis media, COPD & Yes & Yes & Du et al. (1998) \\
\hline \multirow[t]{4}{*}{ Ruminants } & Mannheimia haemolytica & BRD & Yes & & $\begin{array}{l}\text { Ogunnariwo and Schryvers } \\
\text { (1990) }\end{array}$ \\
\hline & Pasteurella multocida & BRD, HS & Yes & & Ogunnariwo et al. (1991) \\
\hline & Histophilus somni & BRD, TME, myocarditis & Yes & & Ogunnariwo et al. (1990) \\
\hline & Bibersteinia trehalosi & Septicemia, pneumomia & Yes & & $\begin{array}{l}\text { Ogunnariwo and Schryvers } \\
\text { (1990) }\end{array}$ \\
\hline Horses & Taylorella equigenitalis & Contagious equine metritis & Yes & Yes & Morgenthau et al. (2012) \\
\hline \multirow[t]{3}{*}{ Pig } & $\begin{array}{l}\text { Actinobacillus } \\
\text { pleuropneumoniae }\end{array}$ & Pleuropneumonia & Yes & & Gonzalez et al. (1990) \\
\hline & Haemophilus parasuis & Glasser's disease & Yes & & Calmettes et al. (2011) \\
\hline & Actinobacillus suis & Pneumonia, septicemia & Yes & & Calmettes et al. (2011) \\
\hline Poultry & $\begin{array}{l}\text { Avibacterium } \\
\text { paragallinarum }\end{array}$ & Infectious coryza & Yes & & $\begin{array}{l}\text { Ogunnariwo and Schryvers } \\
\text { (1992) }\end{array}$ \\
\hline
\end{tabular}

trait it shares with a family of metal iron transport proteins termed TonB-dependent transporters (Tdt) (Cornelissen and Hollander 2011). TonB is part of an inner membrane complex that derives energy from ATP hydrolysis, to support energy requiring outer membrane processes. The ferric ion transported across the outer membrane is transferred to a periplasmic binding protein (ferric binding protein $\mathrm{A}, \mathrm{FbpA}$ ) that delivers the iron atom to an inner membrane transport complex that transports the iron into the cytoplasm (Adhikari et al. 1995).

The Tf and Lf receptors in N. meningitidis and $N$. gonorrhoeae were shown to be specific for the human proteins when they were initially discovered (Schryvers 1988; Schryvers and Morris 1988a, b), providing an explanation for how the host range of these pathogens was restricted to humans. This observation led to the discovery of receptor systems in other Gramnegative pathogens of humans and animals that had specificities that correlated with the host range of the pathogens (Schryvers and Gonzalez 1990), and the exquisite specificity of $\mathrm{Tf}$ receptors from human pathogens was shown to involve binding to a common region of Tf (Gray-Owen and Schryvers 1993). The exquisite host specificity of the $\mathrm{Tf}$ receptor proteins on the human pathogens has been shown to be the result of selective pressures to modify the residues on Tf recognized by the receptor proteins (Barber and Elde 2014), which implies that the Tf receptors have been present for a considerable period of evolutionary history (Fig. 2).

A second type of Tf receptor consisting of a single, smaller Tdt has been identified in Pasteurella multocida (Ogunnariwo et al. 1991) and Histophilus somni (Ekins et al. 2004) but whether it is restricted to bacteria that reside in ruminants or is present in bacteria from other vertebrate hosts is uncertain. The closest homologues are Tdts involved in siderophore or heme iron acquisition and cannot readily be distinguished by standard bioinformatics approaches.

The development of integrated binding and affinity isolation methods led to the discovery of bacterial transferrin $(\mathrm{Tf})$ and lactoferrin (Lf) receptors in the Gram-negative human pathogen Neisseria meningitdis nearly 30 years ago (Schryvers and Morris 1988a, b). The presumed importance of the Tf 

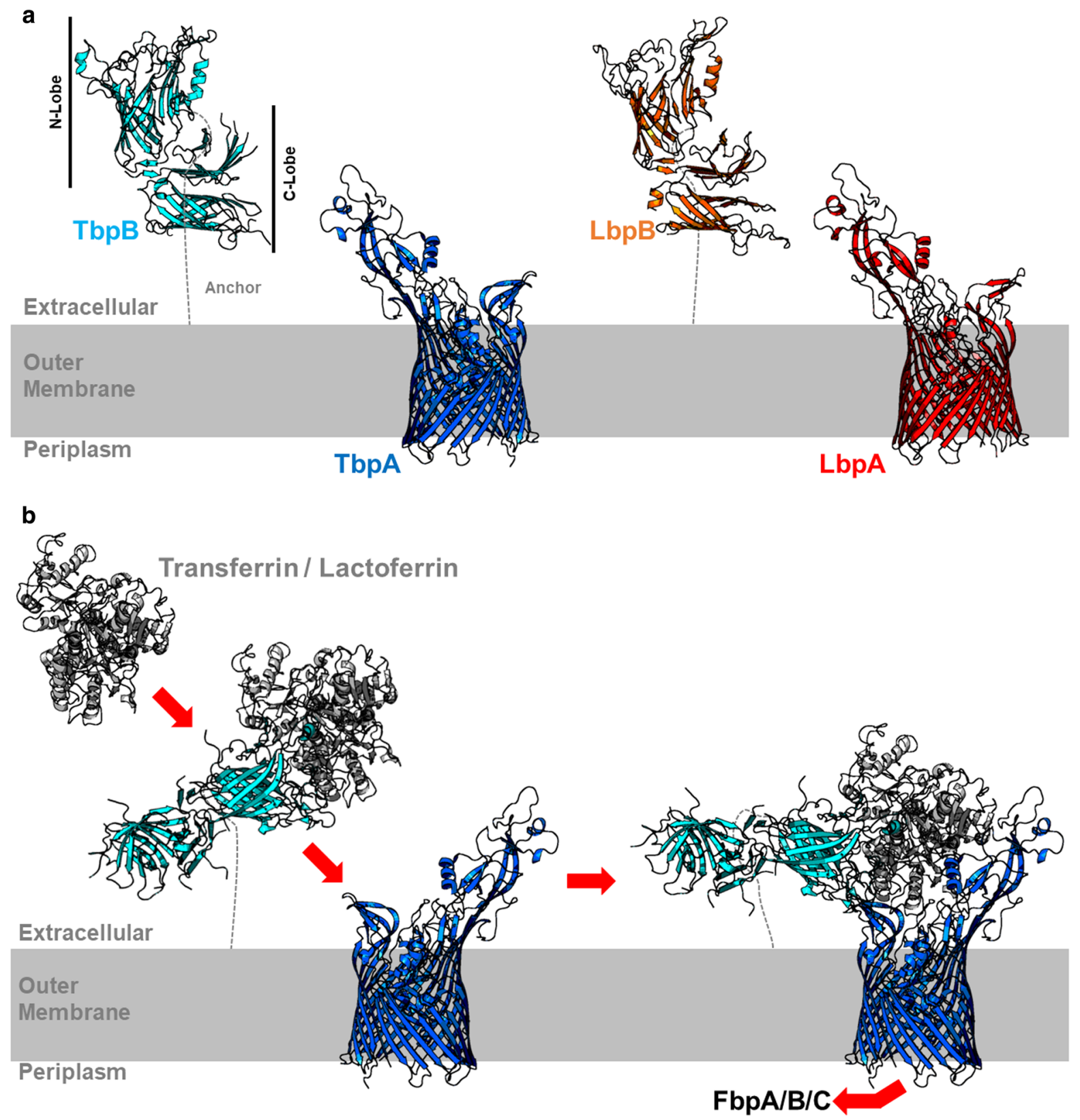

Fig. 1 Transferrin and lactoferrin receptors. Panel a. The bipartite transferrin receptor system comprises of $\mathrm{TbpB}$ (cyan) and TbpA (blue). TbpB is a surface lipoprotein anchored on the extracellular face of the bacterial outer membrane; it has a characteristic bi-lobed structure with a barrel and handle domain in each lobe. TbpA is an integral outer membrane channel, the 22-stranded beta-barrel has a globular plug domain that interacts with the inner membrane TonB complex. The crystal structure of both $\mathrm{TbpB}$ and TbpA in complex with transferrin (Tf) has already been determined (respectively, PDB 3VE2 and 3V8X). The homologous lactoferrin receptor proteins, $\mathrm{LbpB}$ (orange) and $\operatorname{LbpA}$ (red) are predicted to be similar in structure and function to their transferrin receptor counterparts. $\mathrm{LbpB}$ is also a bi-lobed protein but possesses a distinctive charged cluster in the $\mathrm{C}$-lobe that is not present in TbpB. Only the N-lobe crystal structure of $\mathrm{LbpB}$ has been determined to date. Panel b Model for iron acquisition by the transferrin receptor system. The lipid anchored TbpB sequesters iron-loaded host Tf from the extracellular environment and transfers it to TbpA, which removes the iron from the $\mathrm{C}$-lobe of $\mathrm{Tf}$. The iron is translocated across the outer membrane into the periplasm where it is transported into the cell by the ferric binding protein complex (FbpA, B, and C). The lactoferrin receptor system is functionally similar to transferrin receptor complex with $\mathrm{LbpB}$ capturing lactoferrin (Lf) on the cell surface and passing it onto LbpA. (Color figure online) 


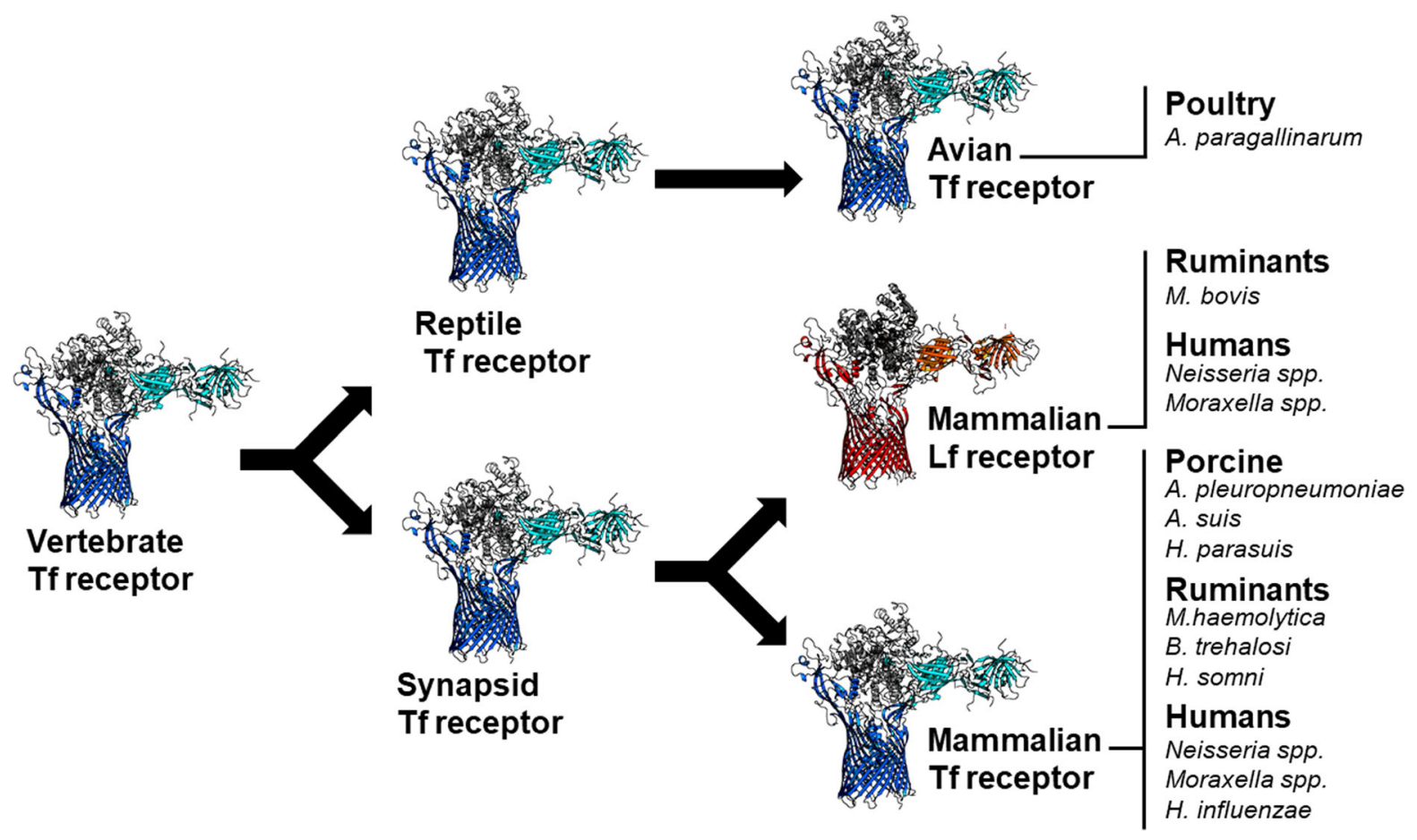

Fig. 2 Proposed ancestral bacterial receptors for the host ferric ion binding glycoproteins, transferrin and lactoferrin. The exquisite host specificity of the bacterial receptors that has

receptors for iron acquisition during invasive infection, supported by the specific requirement for human Tf in a mouse infection model (Schryvers and Gonzalez 1989), and the protection demonstrated in preliminary immunization and challenge experiments in the mouse model (Schryvers 1990), led to focus on the $\mathrm{Tf}$ receptors for development of a group B meningococcal vaccine. Subsequent experiments with the receptor complex (Danve et al. 1993) and the individual TbpB (Tbp2) and TbpA (Tbp1) receptor proteins (Lissolo et al. 1995), isolated from $N$. meningitidis, demonstrated that protection was associated with the TbpB protein, which became the focus of further vaccine development. After a succession of encouraging experiments with recombinant TbpBs in animals (Rokbi et al. 1997), a Phase I clinical trial was performed in humans (Danve et al. 1998), with results that ultimately led to abandoning the commercial development of a TbpB-based vaccine against $N$. meningitidis.

Although the original interest in $\mathrm{Tf}$ and $\mathrm{Lf}$ receptors was focused on their potential utility as vaccine antigens for prevention of invasive infection, these arisen by mutation of host transferrin infers a long-standing relationship between the bacteria and the host as the proposed lineage of evolving receptors illustrates

receptors likely evolved to provide a supply of iron for growth on the mucosal surfaces of the upper respiratory or genitourinary tract which is the normal niche for these bacteria. Not surprisingly, these receptors have been found in pathogens from other vertebrate species as well as some related commensal species that reside in the upper respiratory tract. Since the host specificity of these receptors has evolved over long time periods (Barber and Elde 2014; Barber et al. 2016) (Fig. 2), it is likely they will be found in Gramnegative bacteria from a wide variety of vertebrate hosts while retaining common structural and functional features.

The concept that the $\mathrm{Tf}$ and Lf receptors are important for survival on the mucosal surface is supported by the observation that the $\mathrm{Tf}$ and/or Lf receptors are required for survival of $N$. gonorrhoeae in a human male infection model (Cornelissen et al. 1998; Anderson et al. 2003). Notably, the levels of Tf actually exceed the level of Lf on the mucosal surface of the male urethra prior to challenge with $N$. gonorrhoeae (Anderson et al. 2003), raising the question whether this applies to mucosal surfaces of 
the upper respiratory tract. The observation that mutants in either TbpB or TbpA abrogated infection by Actinobacillus pleuropneumoniae in an aerosol infection model in pigs (Baltes et al. 2002), argues for the presence of $\mathrm{Tf}$ on the surface of the porcine upper respiratory tract. It is challenging to directly measure the levels of $\mathrm{Tf}$ and $\mathrm{Lf}$ on the mucosal surfaces of the upper respiratory tract, thus the strong inferences by studies with mutant bacteria currently provides the best support for this concept. The requirement of $\mathrm{Tf}$ and/or Lf receptors for survival on the mucosal surface argues for their continual expression in this ironrestricted environment, making them suitable targets for vaccines that could prevent colonization, a critical feature for generally non-invasive pathogens like $N$. gonorrhoeae, M. catarrhalis, and non-typeable Haemophilus influenzae.

One advantage of targeting proteins that are common to pathogens from different host species is that they provide the opportunity to address fundamental questions in systems that are more amenable to experimental studies. For instance, it was not possible to directly address the relatively poor performance of the $N$. meningitidis TbpB vaccine in the Phase I trial in humans after such promising results were obtained in the preceding experiments in animals. In order to address the hypothesis that the poor performance was due to host Tf masking important epitopes after systemic administration of $\mathrm{TbpB}$, a non-binding mutant of the TbpB from porcine pathogen Haemophilus parasuis was tested in a lung infection model in pigs (Frandoloso et al. 2015), with the results strongly supporting this hypothesis. Similarly, the observation that the sequence and structural diversity of the TbpBs from three different porcine pathogens was not primarily associated with the geographical region, time of isolation or even the bacterial species (Curran et al. 2015), argued that the diversity primarily reflects protein evolution which developed over a long time-period, suggesting that the development of broadly cross-protective vaccines may be attainable.

Although the preliminary experiments with the receptor proteins isolated from $N$. meningitidis led to the conclusion that $\mathrm{TbpB}$ was predominantly responsible for the protective immune response induced in animals (Lissolo et al. 1995), subsequent experiments demonstrated that functional TbpA was capable of providing protection against infection at least as well as $\mathrm{TbpB}$ and was more effective at inducing a more cross-protective immune response (West et al. 2001). However, functional TbpA has only been obtained by expression in the bacterial outer membrane, providing substantial challenges for production of commercial protein subunit vaccines based on TbpA. This prompted us to develop a strategy of generating hybrid antigens from TbpB and TbpA in which surface epitopes of TbpA are displayed on a scaffold derived from TbpB (Schryvers et al. 2014) which has shown substantial promise in preliminary experiments.

Due to the primary focus on Tf receptors for vaccine development, the $\mathrm{Lf}$ receptors have received relatively little attention. The early observation that $\mathrm{Lf}$ receptors were often absent from strains of $N$. gonorrhoeae (Biswas and Sparling 1995) likely contributed to reduced enthusiasm in targeting lactoferrin receptors for vaccines. The observation that $\mathrm{LbpB}$ may primarily play a role in protection from cationic peptides (Morgenthau et al. 2014) and that LbpB from $N$. meningitidis is released from the surface by proteolytic activity of the NalP autotransporter (Roussel-Jazede et al. 2010) raised questions regarding its utility as a vaccine target. In this study, we re-evaluate the utility of Lf receptors as vaccine targets by examining their prevalence in pathogens and commensal bacteria and assessing the extent to which LbpB is selectively released from the bacterial pathogens expressing this protein.

\section{Materials and methods}

Bacterial strains and growth in iron restriction

M. catarrhalis and $N$. meningitidis strains listed in Table 2 were streaked on chocolate agar plates and were grown overnight at $37{ }^{\circ} \mathrm{C}$ with $5 \% \mathrm{CO} 2$. Colonies from the overnight growth were resuspended in tryptic soy broth (TSB, Becton-Dickinson). Optical densities of the resuspended cells were measured and standardized to an $\mathrm{OD}_{600 \mathrm{~nm}}$ of 0.1 to inoculate $30 \mathrm{~mL}$ of TSB with $30 \mu \mathrm{g} / \mathrm{mL}$ of deferoxamine mesylate (Sigma Aldrich). The cultures were grown shaking at $220 \mathrm{RPM}$ for $5 \mathrm{~h}$ at $37{ }^{\circ} \mathrm{C}$ and the cultures were normalized to an OD600 of 0.75 . 
Isolation of whole cells, outer membrane vesicles, and supernatant

Cell cultures were centrifuged at $3059 \times g$ for $10 \mathrm{~min}$ and the whole cells were re-suspended in $30 \mathrm{~mL}$ of low stringency buffer $(50 \mathrm{mM}$ Tris $\mathrm{pH} 6.0,0.1 \mathrm{M}$ $\mathrm{NaCl}$ ). The spent media were decanted and filtered using $0.2 \mu \mathrm{m}$ Acrodisc $^{\circledR}$ Syringe Filters with Supor ${ }^{\circledR}$ Membrane (Pall) in order to remove any residual cells. The filtered media were then concentrated 30 -folds using 10-kDa cutoff Amicon ${ }^{\circledR}$ Ultra-0.5 mL Centrifugal Filter (Millipore); cOmplete ${ }^{\mathrm{TM}}$ Mini Protease Inhibitor Cocktail tablets (Sigma Aldrich) were added to concentrated media to prevent proteolysis. The concentrated media were centrifuged $(150,000 \times g$, $1 \mathrm{~h})$ to pellet outer membrane vesicles. The supernatants were concentrated 10 -fold using a $10-\mathrm{kDa}$ cutoff Nanosep ${ }^{\circledR}$ centrifugal device (Pall) and the pellets were re-suspended in low stringency buffer, LSB (Bonnah and Schryvers 1998).

Solid-phase binding assay

Samples from the resuspended cells, resuspended ultracentrifuge pellet, and concentrated supernatant were dotted on nitrocellulose membrane (Pall) and was dried at room temperature. The membranes were blocked with $1 \%$ skim milk in LSB for $30 \mathrm{~min}$ at room temperature. The membrane was incubated overnight at $4{ }^{\circ} \mathrm{C}$ with HRP-conjugated hLf/hTf in blocking buffer. The membrane was washed with LSB and then developed in HRP Color Development Reagent (BioRad).

Sample collection and cultivation

Swab samples were obtained from children having their tonsils and adenoids surgically removed at the Alberta Children's Hospital (ACH, Calgary, AB, Canada). Microbiome samples were also obtained directly from the tissue, these samples were diluted and plated on a series of different media incubated under aerobic and anaerobic conditions as described previously (Stearns et al. 2015). Plate pools and individual isolates were collected and stored at $-80{ }^{\circ} \mathrm{C}$ with $20 \%$ skim milk.
Fig. 3 Primary sequence charge window comparisons across LbpBs from six representative strains. The window position on the primary sequence ( $x$-axis) for the 30 -amino acid window is plotted against the resultant net charge ( $y$-axis) of its composite amino acids. EMBOSS charge determines the charge value by simply summing a $(-1)$ charge for acidic residues, a $(+1)$ charge for basic residues, a $(+0.5)$ for histidines, and taking the average using the window width. Negative charge clusters on the plot are outlined with a circle or rectangle. To the right of each charge plot is a three-dimensional model (from SWISSModel) of the respective $\mathrm{LbpB}$ with the $\mathrm{N}$-lobe labelled in white, the $\mathrm{C}$-lobe in blue, and the anionic region in red. (Color figure online)

\section{S rRNA sequencing and genus/species identification}

Individual isolates were used as a template for amplification of a partial 16S rRNA product (900 bp) using primers 8F (AGAGTTTGATC CTGGCTCAG) (Turner et al. 1999) and 926R (CCGTCAATTYYTTTRAGTTT) (Wilmotte et al. 1993). The PCR products were sequenced at Macrogen Inc. (USA). Full 16S rRNA genes from sequenced genomes were located in the contigs and genus/species identification was carried out using Greengenes (http://greengenes.lbl.gov/cgi-bin/nph-index.cgi) and HOMD (http://www.homd.org). A $>98 \%$ sequence identity threshold was used for $16 \mathrm{~S}$ rRNA sequences. The species that could not be discriminated to the species level were identified to the group level.

Illumina MiSeq sequencing

Genomic DNA of selected strains was isolated with a phenol extraction method. The Nextera XT Sample Preparation kit (Illumina) was used to generate libraries and sequenced using the 500 cycle kit on the MiSeq Sequencer (Illumina) running MiSeq Control Software Version 2.4.1.3. Libraries were normalized to $2 \mathrm{nM}$ each and pooled by combining equal volumes of normalized libraries together; the libraries were sequenced on the MiSeq sequencer. The MiSeq analysis folder for the run was input into CASAVA 1.8.2 to determine quality metrics and SPAdes 3.5.0 was used for assembly (Bankevich et al. 2012). 

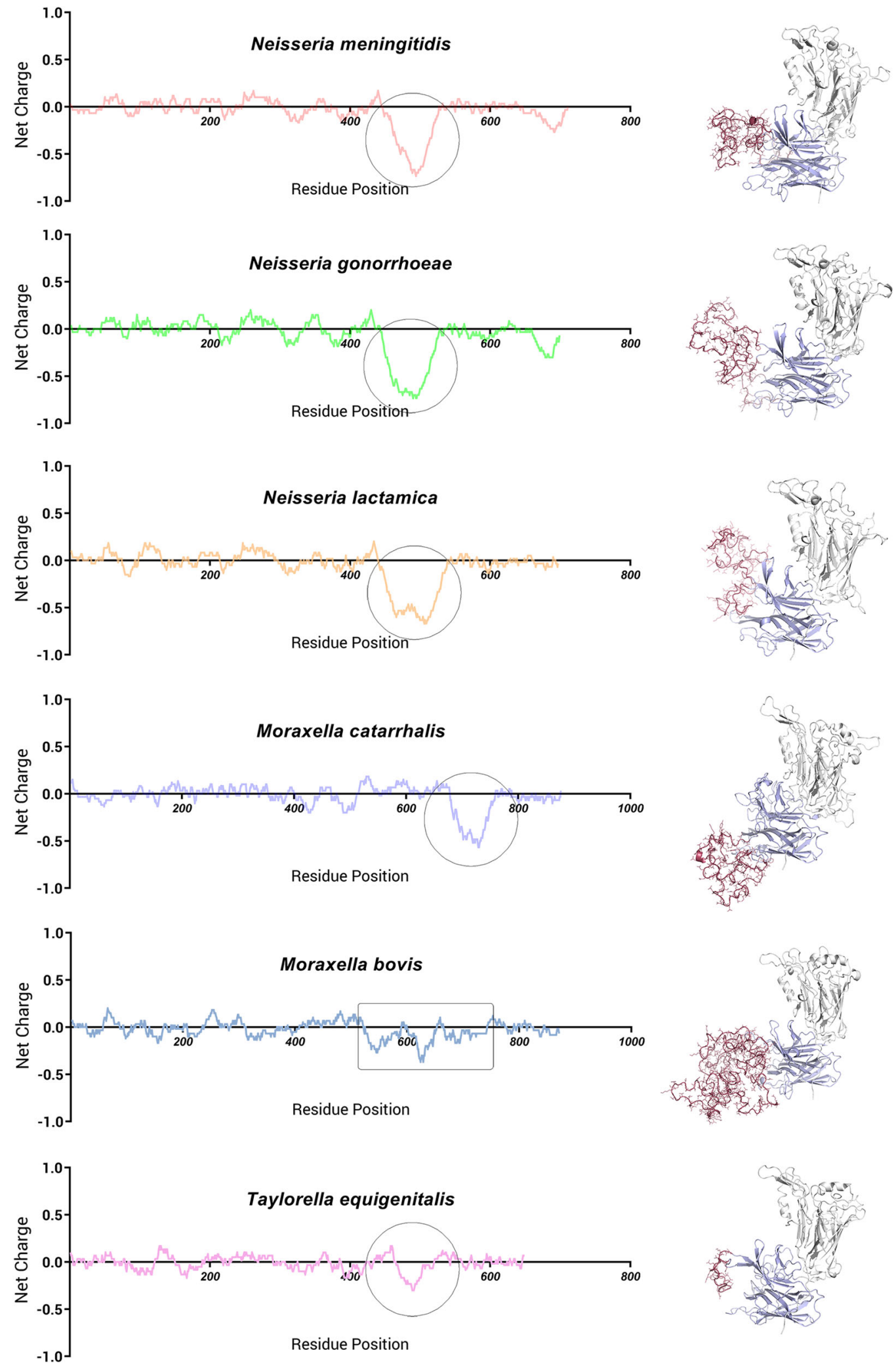
Table 2 Bacterial strains used this study

\begin{tabular}{|c|c|c|}
\hline Bacterial strain & Phenotype & Source \\
\hline M. catarrhalis Q8 & Wildtype & Bergeron MG., Laval University, Quebec, Canada \\
\hline M. catarrhalis No.17 & Wildtype & Ainsworth, S., VAMC, Louisiana, USA \\
\hline M. catarrhalis 4223 & Wildtype & Murphy TF., University at Buffalo SUNY, New York, USA \\
\hline M. catarrhalis $\mathrm{H}-04$ & Wildtype & Campbell GD., Louisiana State University, Louisiana, USA \\
\hline M. catarrhalis $\mathrm{VH}-9$ & Wildtype & Howie V., University of Texas Medical Branch, Texas, USA \\
\hline M. catarrhalis LES1 & Wildtype & $\begin{array}{l}\text { Stenfors LE., Central Hospital of Keski-Pohjanmaa, Kokkola, } \\
\text { Finland }\end{array}$ \\
\hline N. meningitidis MC58 & Wildtype & Tettelin et al. (2000) \\
\hline $\begin{array}{l}\text { N. meningitidis MC58 } \\
\text { nalp::ery }\end{array}$ & $\begin{array}{l}\Delta n a l P \text { with erythromycin } \\
\text { resistance }\end{array}$ & Morgenthau et al. (2014) \\
\hline $\begin{array}{l}\text { N. meningitidis IAL } 2229 \\
\text { (Brazil) }\end{array}$ & Wildtype & Caugant D., Norwegian Institute of Public Health, Norway \\
\hline $\begin{array}{l}\text { N. meningitidis } 860800 \\
\text { (Holland) }\end{array}$ & Wildtype & Caugant D., Norwegian Institute of Public Health, Norway \\
\hline $\begin{array}{l}\text { N. meningitidis } 890326 \\
\text { (Holland) }\end{array}$ & Wildtype & Caugant D., Norwegian Institute of Public Health, Norway \\
\hline
\end{tabular}

\section{Bioinformatics analysis}

NCBI BLASTN and BLASTP were used for identification of homologues of the protein targets in available commensal Neisseria genomes from NCBI (28 genomes), PubMLST database (http://pubmlst. org/neisseria/) (33 genomes) and 9 from our strain collection. To improve quality of analysis and avoid false negatives we only included draft genomes with less than 289 contigs. A threshold of $40 \%$ identity in alignments was used to report the presence or absence of the proteins in Table 3, with the exception of identification of Tbp and Lbp for which higher and true identity was reported in the table.

\section{Modelling of LbpBs}

Three-dimensional models of $\mathrm{LbpB}$ were generated by submitting the primary sequence of each LbpB (obtained from NCBI) to the SWISS-Model server. The server was allowed to automatically search for templates, and in each case, the server selected 3VE1. For each submission, the most complete model of the three server outputs was downloaded and loaded into PyMOL. All models were aligned using the PyMOL align algorithm and lobes/domains were colored accordingly.
Machine learning classifier for LbpB identification

We began classifier training by extracting a large training dataset from NCBI of TbpBs and LbpBs (from all available species) as a way to test how well each of our features contributed to clustering. Only a few features were required to separate the two lipoproteins from each other in feature-space. The features were engineered primarily around a moving-window approach that determined charge clustering of amino acids $\mathrm{K}, \mathrm{R}, \mathrm{H}, \mathrm{D}$, and $\mathrm{E}$ in the same way EMBOSS charge calculates a charge value (Rice et al. 2000). A window size of 40 amino acids was chosen after testing a range of window sizes from 5 to 80 as it negated the noise of a small window yet did not dampen the signal of a large window (Fig. 3a). The EMBOSS charge method of calculating charge clustering turned out to be more informative than isoelectric-point calculations, as the $\mathrm{p} I$ of a negatively charged amino acid cluster will saturate at values close to the $\mathrm{pKa}$ of the amino acids in question, whereas EMBOSS calculates a value that allows for an additive effect of charged amino acids located closely together (Fig. 3b). Using the EMBOSS charge method, we plotted the charge values of 40-amino acid segments for TbpBs and LbpBs, and then determined the maximum 
charge deviation-our first feature-which is the difference between global minimum of the plot and the average total EMBOSS charge of the protein (Fig. 3b, right plot, red line). The relative position in primary sequence $(\mathrm{N}$-terminus $=0, \quad \mathrm{C}$-terminus $=100)$ at which this global minimum occurred was our second feature-global minimum position as LbpBs typically have C-terminal charges. The final two features which proved to be informative as well were simply total isoelectric point and average EMBOSS charge. A scatter-matrix of each of these features plotted against one another can be seen in Fig. 3c. We trained the K-nearest neighbours classifier algorithm $(\mathrm{KNN})$ in the Scikit Learn python machine learning library using a labelled, nonredundant dataset of $123 \mathrm{TbpBs}$ and $59 \mathrm{LbpBs}$ obtained from NCBI-reduced from $\sim 750$ each using the CD-HIT webserver (Huang et al. 2010) which extracts representative sequences from datasets using a $90 \%$ sequence identity threshold. The algorithm is able to distinguish between the two SLPs using primary sequence alone with a $97 \%$ success rate and is freely available upon request.

\section{Results}

Structural features and prevalence of Lf receptor proteins

Relative to the transferrin receptors, there is limited structural information available for the Lf receptors. However, reasonable structural models can be obtained by capitalizing on common features between the two types of receptors (Fig. 1). The considerable sequence homology between TbpA and LbpA, including the highly conserved NEVTGLGCK and GAINEIEYE regions in the plug domain, provides a reasonable model of LbpA based on the structure of the TbpA-hTf complex (Noinaj et al. 2012). It is likely that the external surface loops of the free TbpA and LbpA proteins are in a substantially different (closed) conformation that are altered upon binding of $\mathrm{Tf}$ or $\mathrm{Lf}$, as the binding interaction is proposed to induce separation of the $\mathrm{C} 1$ and $\mathrm{C} 2$ domains of the $\mathrm{C}$-lobe to facilitate iron release. The similarities between TbpA and LbpA make it difficult to definitely distinguish between homologues of the proteins by standard bioinformatics approaches unless there is high sequence identity to a functionally characterized protein. Due to the more extensive prevalence of the Tf receptors, presumably due to the relative importance of $\mathrm{Tf}$ as an iron source on the mucosal surface, $\mathrm{Lf}$ receptors are usually recognized as a second homologue in species that possess $\mathrm{Tf}$ receptors.

To date protein crystallography has yielded structures of the N-terminal domain of LbpB from Moraxella bovis and N. meningitidis (Arutyunova et al. 2012; Brooks et al. 2014) but no structures of the $\mathrm{C}$-lobe or intact protein have been obtained. Crosslinking coupled to mass spectrometry analyses (XLMS) supports the orthogonal orientation of the N-lobe and C-lobe of the N. meningitidis LbpB (Ostan et al. 2017), and it is likely this orientation is preserved in other LbpBs. LbpB\&A can be functionally identified using binding assays, growth assays, and assays measuring the protective effects against cationic peptides associated with the LbpB protein ( $\mathrm{Yu}$ and Schryvers 2002) (Bonnah et al. 1995, 1999; Morgenthau et al. 2012)., with the only distinctive feature of LbpBs being a negatively charged region that is involved in protection against cationic peptides. However, at this juncture it is uncertain whether these regions are invariably present in LbpBs and the lack of sequence identity among these regions, can complicate identification of LbpBs. For instance, the identification a lactoferrin receptor in Taylorella equigenitalis was initially due to detecting two TbpA/LbpA homologues in the species by BLAST searches and then comparing the sequences of the two lipoproteins (Morgenthau et al. 2012). Careful inspection revealed a cluster enriched in acidic amino acids that presumptively identified it as a LbpB.

The identification of a second $\mathrm{Tf} / \mathrm{Lf}$ receptor operon in strains of Actinobacillus pleuropneumoniae (Curran et al. 2015), albeit with a non-functional TbpA/LbpA, is presumed to be $\mathrm{Tf}$ receptor with alternate host specificity due to the absence of a region rich in acidic amino acids. However, without performing functional studies with various Tfs and Lfs, the true identity of this receptor is uncertain. Thus, although it may be relatively straightforward identifying homologues of LbpB and LbpA in closely related species, the discovery of new receptors in unrelated species is not straightforward.

When considering target antigens for vaccines against pathogens, it is important to not only evaluate their prevalence in clinical isolates, but to 
consider their presence in related commensal species that could serve as a reservoir for vaccine escape. Since this also raises questions regarding the consequence of vaccination on the microbiome, it is important to consider whether the different species are potentially dependent upon the target antigen for their survival. Due to extensive study of pathogenic Neisseria species, they provide the best opportunity to address these questions. Thus, we obtained genomic sequences from commensal Neisseria isolates from directed swabs of children undergoing tonsillectomy or adenoidectomy and from our existing strain collection together with sequences available in public databases. We probed the genomes for the presence of $\mathrm{TbpB} \& \mathrm{~A}, \mathrm{LbpB} \& \mathrm{~A}$ and a number of TonB-dependent transporters involved in the acquisition of iron and zinc that have been suggested as potential vaccine targets. These include the hemoglobin/haptoglobin receptor (HpuB/A) (Lewis et al. 1998), the hemoglobin receptor (HmbR) (Stojiljkovic et al. 1996), the ferric enterobactin receptor (FetA) (Carson et al. 2000), the zinc/calprotectin receptor (TdtH) (Jean et al. 2016), and the zinc receptor (ZnuD) (Calmettes et al. 2015). The analysis was extended to include the antigens present in the current Bexsero and Trumemba vaccines so that the issues regarding impact of vaccination on the microbiome and reservoirs for vaccine escape could be compared to existing vaccine products. As illustrated in Table 3, the $\mathrm{LbpB} / \mathrm{A}$ receptors were less prevalent than the $\mathrm{TbpB} / \mathrm{A}$ receptors in the commensal Neisseria species and infrequently found in $N$. flavescens, $N$. subflava, N. sicca and N. mucosa.

Analysis of the N. flavescens, N. subflava, N. sicca and $N$. mucosa genomes reveal that the majority of the TonB dependent transporters are putative siderophore receptors, suggesting that they predominantly rely on pirating siderophores produced by other bacteria, which may be a common feature of microbial communities in diverse ecological niches (D'Onofrio et al. 2010). The limited presence of Lf receptors in these species, and variable presence of Tf receptors, HpuBA and HmbR suggest that they are more likely to encounter $\mathrm{Tf}$ and heme as iron sources than $\mathrm{Lf}$, and might also suggest that they are less likely to encounter host antimicrobial peptides than the other commensal species.
Fig. 4 Feature engineering for TbpB and LbpBs. a Window size of 40 amino acids was selected after testing the signal to noise ratio of several windows on a representative $\mathrm{TbpB}$ and LbpB from Neisseria meningitidis. b Moving window calculations of isoelectric point and EMBOSS charge from a $N$. meningitidis TbpB and LbpB. The average EMBOSS charge is represented by the grey dotted line, and the maximum charge deviation from this average (shown in $\mathrm{LbpB}$ ) is represented with a red line extending from the average to the minimum. $\mathbf{c}$ Scatter matrix of the four selected features and their histograms plotted against each other. TbpBs are labelled in red, and LbpBs in green. Clear clustering in feature space can be seen in many of the plots. (Color figure online)

Features of the negatively charged regions in $\mathrm{LbpBs}$

The negatively charged regions of LbpBs were initially identified by manual inspection. To illustrate their presence, we selected six representative bacterial strains and plotted 30-amino acid segments of the primary sequence as a function of charge, using the European Molecular Biology Open Source Suite (EMBOSS) (Rice et al. 2000) (Fig. 4). The three Neisseria species have relatively large segments of net negative charge reflecting the long stretches that are highly enriched in aspartates and glutamates. The modeled structures preserve the core handle-barrel domains of the N-terminal and $\mathrm{C}$-terminal lobes that are orthogonally positioned, this localizes the clusters of negatively charged domains to a loop in the handle domain of the C-lobe. Notably, in the Neisseria species, there is a second smaller segment of negative charge present in the near the C-terminal end of LbpB for N. meningitidis and N. gonorrhoeae but not in $N$. lactamica, which likely reflects the stretch of 20 amino acids enriched in aspartic acid residues that is positioned in one of the two last loops of the barrel domain. In Moraxella catarrhalis, which has a larger LbpB (900 vs. 700 amino acids in Neisseria spp.), the pattern is similar to that observed in the commensal Neisseria species, but as the structural model illustrates it is likely localized to the first exposed loop of the barrel domain of the C-terminal lobe.

The pattern observed for the Moraxella bovis LbpB is not as obvious as that with the first four LbpBs, reflecting more subtle enrichment of acidic amino acids. This likely is the reason why the negative charge cluster on the $M$. bovis LbpB initially escaped identification via manual examination of the primary 
a

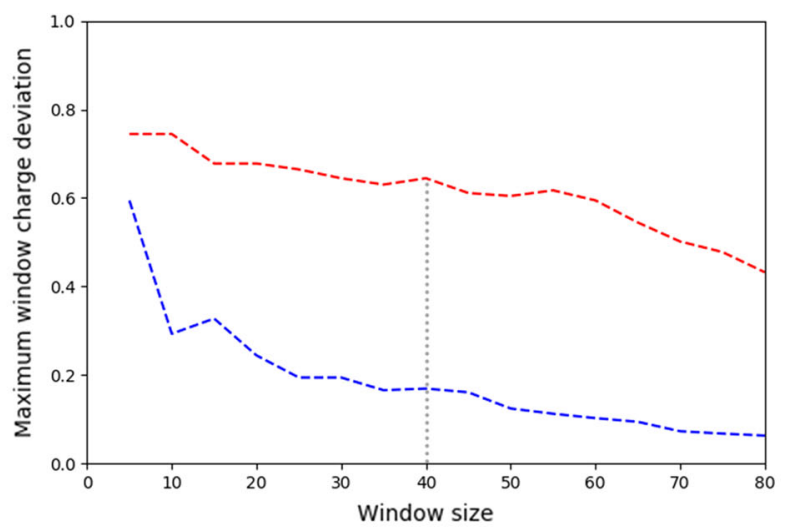

b

Neisseria meningitidis TbpB
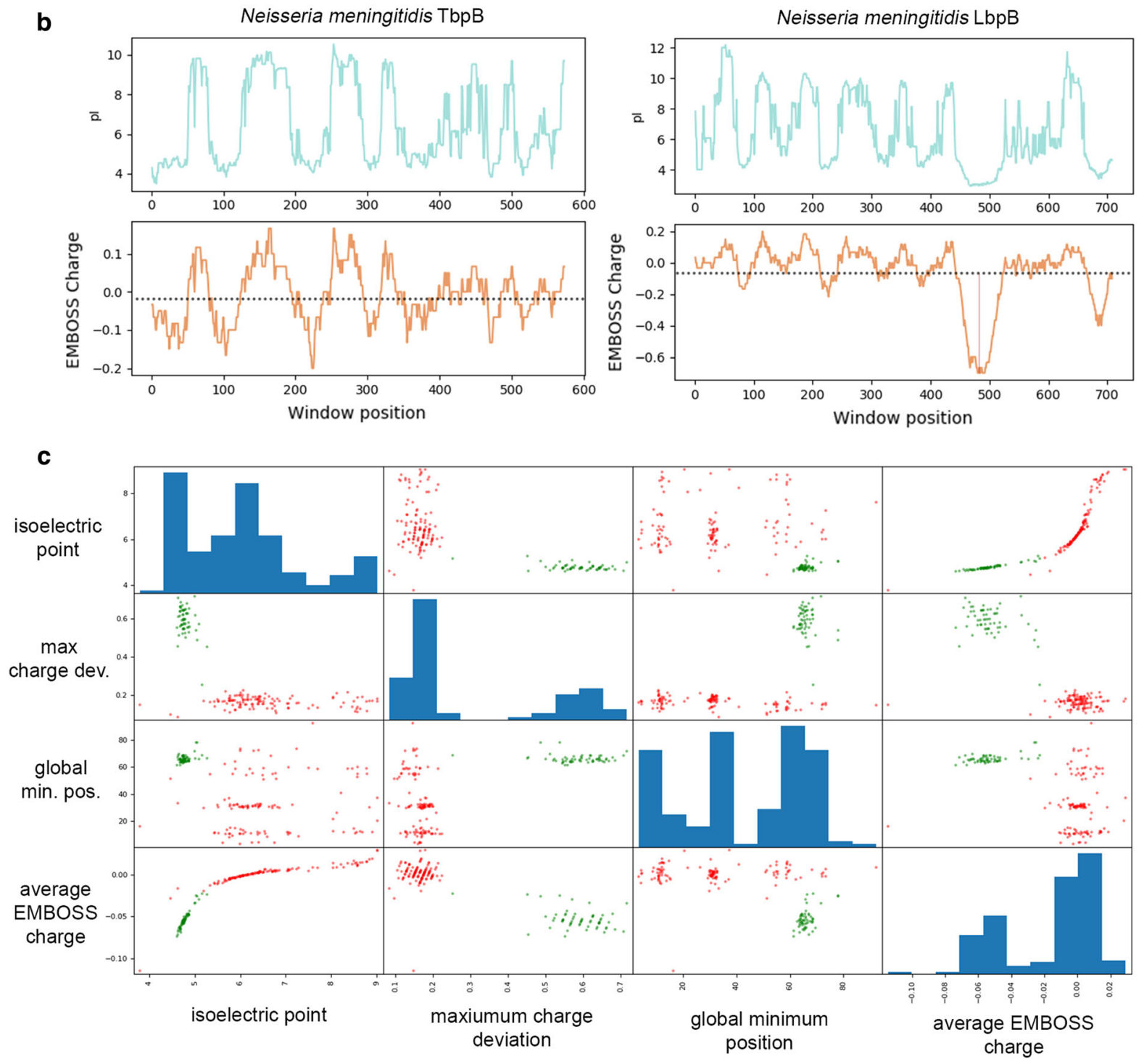
Fig. 5 Solid-phase binding assay of lactoferrin and transferrin binding protein release. Samples from intact whole cells, native outer membrane vesicles (nOMV) preparations, and

concentrated supernatant were dotted on

nitrocellulose membrane and probed with either HRPconjugated hLf or hTf (respectively, left and right columns of figure panels). a $M$. catarrhalis strain Q8 produce a substantially larger amount of nOMVs and increased Lf binding activity in the supernatant fraction is noted. The nalP::ery mutant strain of $N$. meningitidis MC58 has enhanced binding activity for both $\mathrm{Lf}$ and $\mathrm{Tf}$ in both the whole cell and nOMV fractions. b Q8 is compared to five other $M$. catarrhalis strains originating from different geographical regions. Strains Q8, VH-9, and LES-1 released significantly more nOMVs than the other three strains. Lf binding in the supernatant is only detectable in Q8.

c Six meningococcal strains from serogroups A, B, Y, and $\mathrm{Z}$ were compared and all strains produced detectable quantities of nOMVs based on Tf binding a

HRP-hLf

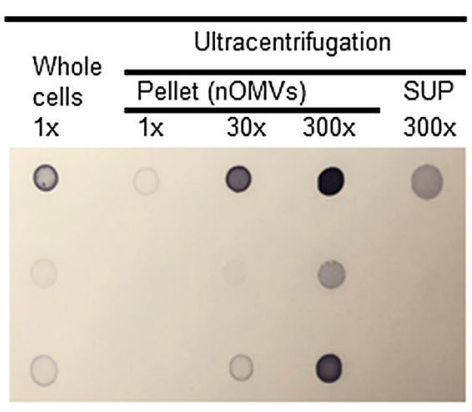

b

Mic No. 17

Mc 4223

Mic H-04

Mic Q8

NG VH-9

Mic LES1

c

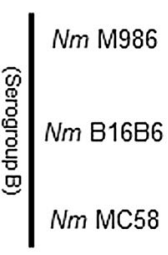

Nm IAL2229

(Serogroup A)

Nm 860800

(Serogroup $Y$ )

Nm 890326

(Serogroup Z)
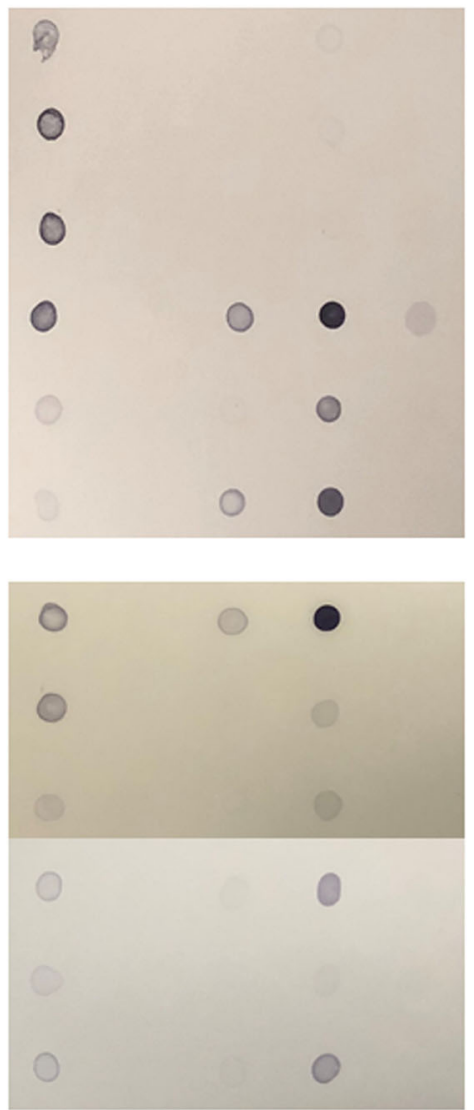

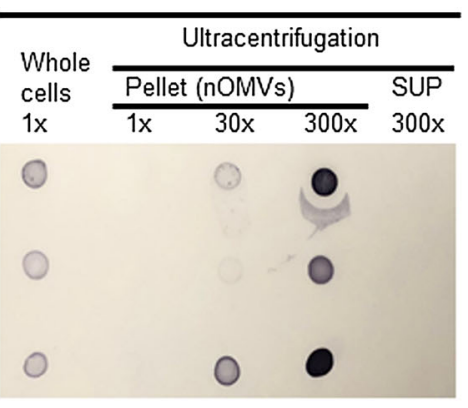

HRP-hTf
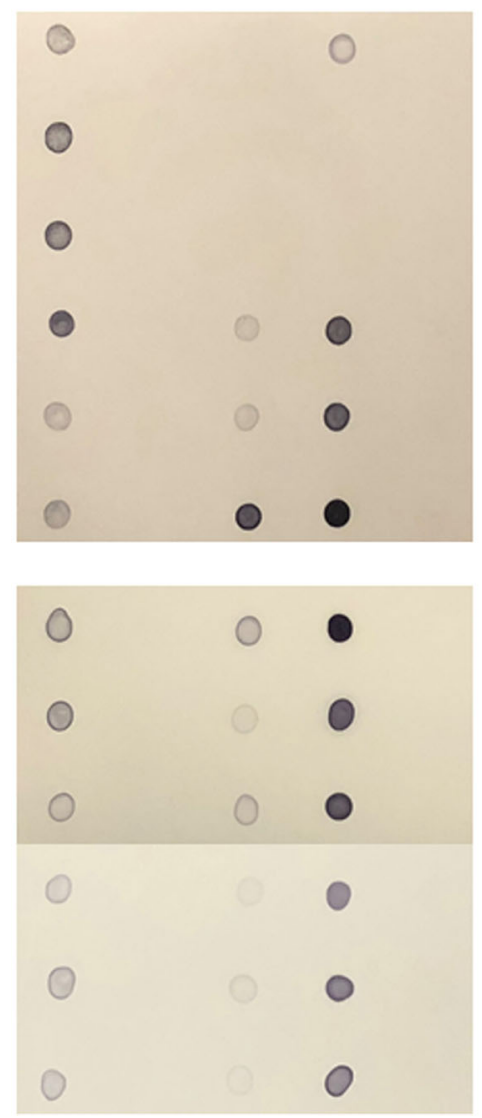

protein sequence/isoelectric point calculations in a moving window approach. Using more advanced bioinformatic methods to model this LbpB, we found the acidic region to be present within amino acid stretches extending from one of the C-lobe handle strands. Negatively charged amino acids in this protein are distributed more sparsely and likely to localize within 3-dimensional space. The pattern observed for Taylorella equigenitalis $\mathrm{LbpB}$ is also subtler than that observed for the first four LbpBs (attributable to its smaller size), and was originally noticed by comparisons between the two lipoproteins associated with the putative Tf and Lf TonB-dependent transporter genes. It has a smaller cluster of acidic amino acids comparable to those present in the in the Neisseria species and this cluster appears to be localized to the handle domain of the C-lobe.

Although identifying putative LbpBs using manual sequence curation has been successful in the past, some important features of these lipoproteins have 
Table 3 Prevalence of surface antigens in commensal Neisseria species

\begin{tabular}{|c|c|c|c|c|c|}
\hline \multirow[t]{2}{*}{ Species (\# of strains) } & \multicolumn{5}{|c|}{ Percentage of strains with the indicated protein target } \\
\hline & TbpBA & LbpBA & HрuAB & $\mathrm{HmbR}$ & $\underline{\text { FetA }}$ \\
\hline N. lactamica (10) & 100 & 100 & 100 & 0 & 100 \\
\hline N. polysacchareae (10) & 100 & 50 & 100 & 10 & 100 \\
\hline N. cineria $(10)$ & 100 & 100 & 90 & 10 & 100 \\
\hline N. flavescens/subflava (20) & 30 & 5 & 45 & 50 & $65+10^{*}$ \\
\hline N. sicca/mucosa (10) & 40 & 10 & 50 & 40 & $60+40^{*}$ \\
\hline \multirow[t]{2}{*}{ Species (\# of strains) } & \multicolumn{5}{|c|}{ Percentage of strains with the indicated protein target } \\
\hline & fHbp & $\mathrm{NadA}$ & NHBA & TdfH & ZnuD \\
\hline N. lactamica (10) & 0 & 0 & 100 & 50 & 100 \\
\hline N. polysacchareae (10) & 100 & 0 & 100 & 0 & 100 \\
\hline N. cineria $(10)$ & $90+10^{*}$ & $30+20 *$ & 10 & 70 & 100 \\
\hline N. flavescens/subflava (20) & $80^{*}$ & 0 & 20 & 100 & 75 \\
\hline N. sicca/mисоsa (10) & $40^{*}$ & $30 *$ & 0 & 80 & 100 \\
\hline
\end{tabular}

NCBI BLASTN and BLASTP were used for identification of homologues of surface antigens in available commensal Neisseria genomes from NCBI,PubMLST database and the lab collection. NadA (Neisseria adhesion A), NHBA (Nesserial Heparin Binding Antigen), TdfH (TonB-dependent family $\mathrm{H}$ ). Prevalence of target proteins presented in percentage

*Indicates $40-50 \%$ identity to the query

been overlooked in cases where the charge clustering is less obvious than in LbpBs from Neisseria spp. Additionally, with increasing amounts of genomic data being deposited in public databases such as NCBI, manually identifying LbpBs from libraries of strains for purposes such as vaccine antigen selection is a time-consuming and inefficient approach. We thus sought to train a machine-learning classifier using features from existing LbpBs that could extract these proteins from a genomic annotation with a high degree of accuracy and the exclusion of human bias. The classifier takes advantage of the fact that LbpBs have C-terminal negative charge clusters. After training the classifier, we tested its ability to identify more difficult LbpBs, and found it was able to identify the Taylorella equigenitalis $\mathrm{LbpB}$, just as was done previously by manual inspection. The classifier is also able to differentiate a TbpB from an $\mathrm{LbpB}$ with a $97 \%$ success rate in a variety of other species. Figure $3 \mathrm{~b}$ and c illustrate the main features which were engineered to distinguish a TbpB from an LbpB, and their clustering in feature-space (more detailed explanation available in methods section). To examine if our classifier was able to identify new LbpBs, we retrieved genomic contigs from Moraxella lacunata from NCBI and annotated the genomic assembly using RAST (Aziz et al. 2008). The classifier successfully identified one previously undocumented LbpB which we confirmed using manual sequence inspection. The classifier, which utilizes Scikit-learn/Biopython libraries as well as custom scripts is available upon request for personal use.

\section{Release of $\mathrm{LbpB}$}

The reported selective release of the Neisseria meningitidis $\mathrm{LbpB}$ from the cell surface by the proteolytic activity of the autotransporter NalP, with a concomitant decrease in killing in a bactericidal assay (Roussel-Jazede et al. 2010), has important implications regarding its suitability as a vaccine target. This prompted us to evaluate whether this phenomenon was also observed with LbpBs from different species. Initially we performed an experiment to compare a strain of $M$. catarrhalis (Q8) to a wild-type strain of N. meningitidis (MC58) and a NalP deficient derivative (Fig. 5A). We assessed the Lf binding activity of intact cells, native outer membrane vesicle preparations and supernatants from these three strains, and also evaluated the Tf binding activity as a 
control. As shown in panel A of Fig. 5, the nalP::ery mutant strain surprisingly had enhanced binding activity in the solid binding assay both with labeled Lf and Tf on intact cells and the outer membrane vesicle fraction. A logical inference is that the proteolytic activity of NalP may be associated with the release of both $\mathrm{LbpB}$ and $\mathrm{TbpB}$ from the cell surface, suggesting that the release of lipoproteins by NalP may not be specific.

The lack of detectable Lf binding activity in the supernatant fraction from the wild-type meningococcal cells at 300 times the concentration of the cell suspension was unexpected based on the results from previous studies (Roussel-Jazede et al. 2010). Our inability to detect Lf binding activity in dot assays or to affinity capture LbpB from the supernatants (data not shown) could be due to loss of protein during manipulations in spite of inclusion of protease inhibitors. Nevertheless, the reduced binding observed in the nalP::ery mutant strain is consistent with previous studies and questions regarding the release of TbpB or other lipoproteins requires further investigation.

The relatively prolific production of nOMVs by $M$. catarrhalis strain Q8 and Lf binding activity in the supernatant fraction prompted us to examine a set of M. catarrhalis strains from different geographical regions to see whether these phenomena were present in other M. catarrhalis strains (Fig. 5B). Notably there was substantial release of nOMVs by 3 of the 6 selected strains (Q8, VH-9 and LES1) whereas release of nOMVs by the other three strains was not detected or was at least 10-fold less. However, strain Q8 was to only strain with detectable binding of Lf by the supernatant fraction, and it will require more direct experimental evidence to suggest it is due to released LbpB.

In order to make a more general comparison between strains of $M$. catarrhalis and $N$. meningitidis, we also selected a panel of six meningococcal strains with coverage from serogroups $\mathrm{A}, \mathrm{B}, \mathrm{Y}$, and $\mathrm{Z}$ (Fig. 5c). In contrast to M. catarrhalis, the meningococcal strains all produced substantial quantities of nOMVs based on Tf binding. Notably, the pattern of Lf binding by strain M986 resembled that of the nalP::ery mutant, suggesting that this strain or isolate may be NalP deficient.

\section{Discussion}

Although there are a variety of approaches for selecting appropriate target antigens for vaccines, perhaps the most compelling is selection of an antigen that is essential for survival and disease causation as it provides the potential for eliminating the disease and excludes the potential for vaccine escape by loss of the target antigen. Using this rationale for the diseases listed in Table 1, the target antigen that best meets these criteria is the transferrin receptor proteins present in the pathogens from the family Pasteurellaceae, in which both TbpB and TbpA have been shown to be essential for survival and disease causation by the porcine pathogen Actinobacillus pleuropneumoniae (Baltes et al. 2002). A structure-based design approach was used to develop a binding deficient derivative of $\mathrm{TbpB}$ that was shown to be very effective at prevention of Glasser's disease by the porcine pathogen Haemophilus parasuis (Frandoloso et al. 2015). The $\mathrm{Tf}$ receptor proteins are common to the three porcine pathogens listed in Table 1, and analysis of the sequence and structural diversity of these proteins indicated that it was not restricted to species, geographical region or time of isolation, indicating that the phylogenetic clustering is a reflection of protein evolution (Curran et al. 2015), and suggests that a Tbp-based vaccine could be developed to target all three porcine pathogens. It is important to recognize that in bacterial species with highly efficient natural transformation systems such as those integral to members of the Pasteurellacea (Redfield et al. 2006), antigenic variation mechanisms primarily rely on efficient uptake of variant genes rather than new variants arising by mutation.

In spite of considerable sequence diversity in the TbpBs, the prospects of developing an effective and broadly cross-protective vaccine derived from $\mathrm{TbpBs}$ are good, and since $\mathrm{TbpB}$ was shown to be essential for survival and disease causation (Baltes et al. 2002), the prospect for vaccine escape by loss of TbpB seems unlikely. Since the upper respiratory or genitourinary tract of the vertebrate host is the only ecological niche for these Gram-negative bacteria, development of an effective immune response that would reduce or prevent colonization could potentially eliminate these pathogens from their vertebrate host. Although the bipartite TbpB-TbpA receptor has been preserved in bacteria present in vertebrate hosts ranging from 
poultry to humans (Fig. 2), the single protein TbpA2 receptor present in the bovine pathogens Pasteurella multocida and Histophilus somni demonstrate that an accessory lipoprotein is not always essential for iron acquisition from host transferrin. The prevalence of TbpA2 among vertebrates is uncertain since the closest homologues are TonB-dependent transporters involved in heme and siderophore uptake and TbpA2 s would only be recognized by functional assays. Since the TbpA and TbpA 2 proteins are responsible for the actual transport of iron across the outer membrane and have substantially less sequence variation, they are potentially more ideal vaccine targets. However, it would be challenging to develop protein subunit vaccines with the TbpA and TbpA2 integral outer membrane proteins since production of functional protein requires insertion and assembly in the outer membrane and extraction with detergents.

The demonstration that either the lactoferrin receptor or the transferrin receptor could support the growth and survival of $N$. gonorrhoeae in the human male genitourinary tract (Anderson et al. 2003), and its invariant presence in the pathogens that primarily reside in the upper respiratory tract (Table 1), suggest that it is also a good target antigen for vaccines against these pathogens. As with TbpA, LbpA is an attractive target but challenges in its commercial production would need to be addressed. The identification of regions enriched in negatively charged amino acids in LbpB and the subsequent demonstration that they are involved in protection against cationic antimicrobial peptides (Morgenthau et al. 2012, 2014), suggest that its primarily role may no longer be related to iron acquisition. However, recent studies suggest that LbpB is capable of binding Lf in both its N-terminal and C-terminal lobes (Ostan et al. 2017), thus LbpB may be capable of fulfilling both roles, depending upon conditions such as iron status and concentration of Lf. It is interesting to note that, in contrast to $\mathrm{Tf}$ where positive selection is driving changes in the TbpA binding residues of Tf (Barber and Elde 2014), positive selection is primarily driving changes in the positively charged $\mathrm{N}$-terminal region of lactoferrin (Barber et al. 2016) (Liang and Jiang 2010).

The report of release of the $N$. meningitidis LbpB from the cell surface by proteolytic activity of the NalP autotransporter protein raised additional concerns regarding its potential as a target for vaccination (Roussel-Jazede et al. 2010), with both the released
LbpB potentially competing for antibody and the reduced target on the cell surface affecting antibodymediated killing. Our results (Fig. 5, top panel) suggest that $\mathrm{TbpB}$ is also being released from the cell surface by NalP, which raises the question whether it can mediate the release of other lipoproteins such as fHbp. Additionally, it is uncertain what the expression levels of NalP will be under different conditions in vivo, and how that might influence the immune effector mechanisms. Thus, on the normal mucosal surface where there may be a substantial proportion of iron-loaded Lf, the release of LbpB would not be advantageous and expression of NalP may not be favored, while under inflammatory conditions where Lf is predominantly in the apo form, accompanied by the cationic peptides from the neutrophil and release from Lf, release of LbpB by NalP activity could be favored (Ostan et al. 2017). Challenges in detecting released $\mathrm{LbpB}$ by our solid-phase binding assay prevented us from determining how widespread this phenomenon is, and although preliminary evidence might suggest that it also occurs in $M$. catarrhalis (Fig. 5, top row), further experiments would be required to confirm this.

In this study, we also evaluated the binding activity associated with native outer membrane vesicles (nOMVs) which provided an indication of the relative amount of released membrane to intact cells (Fig. 5). The results indicate that there is considerable strain to strain variation in $M$. catarrhalis in regards to the release of nOMVs, with some strains not producing detectable nOMVs under laboratory conditions. In most instances, the binding activity associated with nOMVs is between 1/10th and 1/30th of the level present in intact cells, which suggest that simple immune evasion by competing for antibody binding with intact cells would not be very effective at the levels of membrane blebbing occurring at levels observed under in vitro conditions. The observation that nOMVs from Opa-expressing $N$. meningitidis bound CEACAM1(carcinoembryonic antigen-related cellular adhesion molecules) and suppressed activation of CD4+T-lymphocytes provides one example of more complex activities the nOMVs could mediate by diffusion into tissues surrounding the bacteria (Lee et al. 2007). The nOMVs from M. catarrhalis have been proposed to facilitate degradation of beta-lactam antibiotics (Schaar et al. 2011) and reducing complement-mediated killing, but how effective these 
mechanisms would occur in vivo depend on the levels of production.

The prevalence of target antigens in related commensal bacteria have two important ramifications (i) the potential to serve as a reservoir for vaccine escape if they represent variants not covered by the vaccine, and (ii) the potential of the vaccine to impact the commensal flora if the vaccine can impact mucosal colonization as has been observed with the conjugate capsular vaccines (Maiden et al. 2008; Kellner et al. 2008). Based on the inherent charge characteristics of the LbpB receptor (Fig. 3), we have developed a machine learning classifier that will identify and differentiate putative Lf and $\mathrm{Tf}$ receptors (Fig. 4). This classifier algorithm can be applied to genomic data of related commensal bacteria and identify receptor variants that need to be considered for potential vaccine escape. In regards to the impact on commensal flora one should consider how important the target antigen is for survival, as commensal flora could escape the impact of vaccination by loss of the target since the receptor may not be critical for survival. Thus, in various commensal Neisseria species the $\mathrm{Tf}$ and Lf receptors range from being invariably present to only occasionally being present (Table 3). Thus, in contrast to pathogenic Neisseria species that rely on $\mathrm{Tf}$ and $\mathrm{Lf}$ receptors for survival (Anderson et al. 2003), some commensal species primarily rely on other iron acquisition mechanisms, such as scavenging iron with siderophores produced by other microbes, thus would be minimally impacted by vaccination (Table 3 ). It is uncertain what the impact would be on commensal species that normally possess $\mathrm{Tf}$ and Lf receptors, but they generally seem to have a larger repertoire of siderophore receptors than the pathogenic species and are less likely to be dependent on the $\mathrm{Tf}$ and $\mathrm{Lf}$ receptors for survival. $\mathrm{Lf}$ receptors appear to be less prevalent than $\mathrm{Tf}$ receptors among the commensal species, thus targeting the Lf receptors might have less impact on the commensal flora and might be less susceptible to vaccine escape by the reservoir in commensals. In balance, Lf receptors are viable vaccine targets and are worth exploring as such.

Acknowledgements This work was supported by funding from the Canadian Institutes of Health Research (Grant MOP138273), and the National Sciences and Engineering Council (RGPIN-2016-04555) for ABS.
Funding This study was supported by the Canadian Institutes of Health Research (Grant Number MOP138273) and the National Sciences and Engineering Council of Canada (Discovery Grant 298351-2010).

\section{Compliance with ethical standards}

Conflict of interest $\mathrm{ABS}$ is a stakeholder in Engineered Antigens Inc.

\section{References}

Adhikari P, Kirby SD, Nowalk AJ, Veraldi KL, Schryvers AB, Mietzner TA (1995) Biochemical characterization of a Haemophilus influenzae periplasmic iron transport operon. J Biol Chem 42:25142-25149

Anderson JE, Hobbs MM, Biswas GD, Sparling PF (2003) Opposing selective forces for expression of the gonococcal lactoferrin receptor. Mol Microbiol 48(5):1325-1337

Arutyunova E, Brooks CL, Beddek A, Mak MW, Schryvers AB, Lemieux MJ (2012) Crystal structure of the N-lobe of lactoferrin binding protein B from Moraxella bovis. Biochem Cell Biol 90(3):351-361. https://doi.org/10.1139/ o11-078

Aziz RK, Bartels D, Best AA, DeJongh M, Disz T, Edwards RA, Formsma K, Gerdes S, Glass EM, Kubal M, Meyer F, Olsen GJ, Olson R, Osterman AL, Overbeek RA, McNeil LK, Paarmann D, Paczian T, Parrello B, Pusch GD, Reich C, Stevens R, Vassieva O, Vonstein V, Wilke A, Zagnitko O (2008) The RAST server: rapid annotations using subsystems technology. BMC Genom 9:75. https://doi.org/10. 1186/1471-2164-9-75

Baltes N, Hennig-Pauka I, Gerlach GF (2002) Both transferrin binding proteins are virulence factors in Actinobacillus pleuropneumoniae serotype 7 infection. FEMS Microbiol Lett 209(2):283-287

Bankevich A, Nurk S, Antipov D, Gurevich AA, Dvorkin M, Kulikov AS, Lesin VM, Nikolenko SI, Pham S, Prjibelski AD, Pyshkin AV, Sirotkin AV, Vyahhi N, Tesler G, Alekseyev MA, Pevzner PA (2012) SPAdes: a new genome assembly algorithm and its applications to single-cell sequencing. J Comput Biol 19(5):455-477. https://doi.org/ $10.1089 / \mathrm{cmb} .2012 .0021$

Barber MF, Elde NC (2014) Nutritional immunity. Escape from bacterial iron piracy through rapid evolution of transferrin. Science 346(6215):1362-1366. https://doi.org/10.1126/ science. 1259329

Barber MF, Kronenberg Z, Yandell M, Elde NC (2016) Antimicrobial functions of lactoferrin promote genetic conflicts in ancient primates and modern humans. PLoS Genet 12(5):e1006063. https://doi.org/10.1371/journal. pgen. 1006063

Biswas GD, Sparling PF (1995) Characterization of lbpA, the structural gene for a lactoferrin receptor in Neisseria gonorrhoeae. Infect Immun 63(8):2958-2967

Bonnah RA, Schryvers AB (1998) Preparation and characterization of Neisseria meningitidis mutants deficient in the production of the human lactoferrin binding proteins LbpA and LbpB. J Bacteriol 180(12):3080-3090 
Bonnah RA, Yu R-H, Schryvers AB (1995) Biochemical analysis of lactoferrin receptors in the Neisseriaceae: identification of a second bacterial lactoferrin receptor protein. Microb Pathog 19(5):285-297

Bonnah RA, Wong H, Loosmore SM, Schryvers AB (1999) Characterization of Moraxella (Branhamella) catarrhalis $l b p B, l b p A$ and lactoferrin receptor orf 3 isogenic mutants. Infect Immun 67(3):1517-1520

Brooks CL, Arutyunova E, Lemieux MJ (2014) The structure of lactoferrin-binding protein B from Neisseria meningitidis suggests roles in iron acquisition and neutralization of host defences. Acta Crystallograph Sect F 70(Pt 10):1312-1317. https://doi.org/10.1107/S2053230X14019372

Calmettes C, Yu R-H, Silva LP, Curran D, Schriemer DC, Schryvers AB, Moraes TF (2011) Structural variations within the transferrin binding site on transferrin binding protein, TbpB. J Biol Chem 286:12683-12692

Calmettes C, Ing C, Buckwalter CM, El Bakkouri M, Chieh-Lin Lai C, Pogoutse A, Gray-Owen SD, Pomes R, Moraes TF (2015) The molecular mechanism of Zinc acquisition by the neisserial outer-membrane transporter ZnuD. Nat Commun 6:7996. https://doi.org/10.1038/ncomms8996

Carson SD, Stone B, Beucher M, Fu J, Sparling PF (2000) Phase variation of the gonococcal siderophore receptor FetA. Mol Microbiol 36(3):585-593

Cornelissen CN, Hollander A (2011) TonB-dependent transporters expressed by Neisseria gonorrhoeae. Front Microbiol 2:117. https://doi.org/10.3389/fmicb.2011.00117

Cornelissen CN, Kelley M, Hobbs MM, Anderson JE, Cannon JG, Cohen MS, Sparling PF (1998) The transferrin receptor expressed by gonococcal strain FA1090 is required for the experimental infection of human male volunteers. Mol Microbiol 27(3):611-616

Curran D, Adamiak P, Fegan J, Qian C, Yu R, Schryvers AB (2015) Sequence and structural diversity of transferrin receptors in Gram-negative porcine pathogens. Vaccine 33(42):5700-5707

Danve B, Lissolo L, Mignon M, Dumas P, Colombani S, Schryvers AB, Quentin-Millet MJ (1993) Transferrinbinding proteins isolated from Neisseria meningitidis elicit protective and bactericidal antibodies in laboratory animals. Vaccine 11:1214-1220

Danve B, Lissolo L, Guinet F, Boutry E, Speck D, Cadoz M, Nassif X, Quentin-Millet MJ (1998) Safety and immunogenicity of a Neisseria meningitidis group B transferrin binding protein vaccine in adults. In: Nassif X, QuentinMillet M-J, Taha M-K (eds) 11th International Pathogenic Neisseria Conference, Nice, 1998. Eleventh International Pathogenic Neisseria Conference

D’Onofrio A, Crawford JM, Stewart EJ, Witt K, Gavrish E, Epstein S, Clardy J, Lewis K (2010) Siderophores from neighboring organisms promote the growth of uncultured bacteria. Chem Biol 17(3):254-264. https://doi.org/10. 1016/j.chembiol.2010.02.010

Du R, Wang Q, Yang Y-P, Schryvers AB, Chong P, England D, Klein MH, Loosmore SM (1998) Cloning and expression of the Moraxella catarrhalis lactoferrin receptor genes. Infect Immun 66(8):3656-3664

Ekins A, Bahrami F, Sijercic A, Maret D, Niven DF (2004) Haemophilus somnus possesses two systems for acquisition of transferrin-bound iron. J Bacteriol 186(13):4407-4411
Frandoloso R, Martinez-Martinez S, Calmettes C, Fegan J, Costa E, Curran D, Yu R, Gutierrez-Martin CB, RodriguezFerri EF, Moraes TF, Schryvers AB (2015) Nonbinding site-directed mutants of transferrin binding protein $\mathrm{B}$ enhances their immunogenicity and protective capabilities. Infect Immun 83(3):1030-1038

Gonzalez GC, Caamano DL, Schryvers AB (1990) Identification and characterization of a porcine-specific transferrin receptor in Actinobacillus pleuropneumoniae. Mol Microbiol 4:1173-1179

Gray-Owen SD, Schryvers AB (1993) The interaction of primate transferrins with receptors on bacteria pathogenic to humans. Microb Pathog 14:389-398

Gray-Owen SD, Schryvers AB (1995) Characterization of transferrin binding proteins 1 and 2 in invasive type $\mathrm{b}$ and nontypable strains of Haemophilus influenzae. Infect Immun 63(10):3809-3815

Huang Y, Niu B, Gao Y, Fu L, Li W (2010) CD-HIT Suite: a web server for clustering and comparing biological sequences. Bioinformatics 26(5):680-682. https://doi.org/ 10.1093/bioinformatics/btq003

Jean S, Juneau RA, Criss AK, Cornelissen CN (2016) Neisseria gonorrhoeae evades calprotectin-mediated nutritional immunity and survives neutrophil extracellular traps by production of TdfH. Infect Immun 84(10):2982-2994. https://doi.org/10.1128/IAI.00319-16

Kellner J, Scheifele D, Vanderkooi O, MacDonald J, Church D (2008) Effects of routine infant vaccination with the 7-valent pneumococcal conjugate vaccine on nasopharyngeal colonization with streptococcus pneumoniae in children in Calgary, Canada. Pediatr Infect Dis J 27:526-532

Lee HS, Boulton IC, Reddin K, Wong H, Halliwell D, Mandelboim O, Gorringe AR, Gray-Owen SD (2007) Neisserial outer membrane vesicles bind the co-inhibitory receptor CEACAM1 and suppress CD4+T lymphocyte function. Infect Immun 75:4449-4455

Lewis LA, Sung MH, Gipson M, Hartman K, Dyer DW (1998) Transport of intact porphyrin by $\mathrm{HpuAB}$, the hemoglobinhaptoglobin utilization system of Neisseria meningitidis. J Bacteriol 180(22):6043-6047

Liang GM, Jiang XP (2010) Positive selection drives lactoferrin evolution in mammals. Genetica 138(7):757-762. https:// doi.org/10.1007/s10709-010-9456-x

Lissolo L, Maitre-Wilmotte G, Dumas P, Mignon M, Danve B, Quentin-Millet M-J (1995) Evaluation of transferrinbinding protein 2 within the transferrin- binding protein complex as a potential antigen for future meningococcal vaccines. Infect Immun 63(3):884-890

Maiden MC, Ibarz-Pavon AB, Urwin R, Gray SJ, Andrews NJ, Clarke SC, Walker AM, Evans MR, Kroll JS, Neal KR, Ala'aldeen DA, Crook DW, Cann K, Harrison S, Cunningham R, Baxter D, Kaczmarski E, Maclennan J, Cameron JC, Stuart JM (2008) Impact of meningococcal serogroup $\mathrm{C}$ conjugate vaccines on carriage and herd immunity. J Infect Dis 197(5):737-743. https://doi.org/10. 1086/527401

Morgenthau A, Livingstone M, Adamiak P, Schryvers AB (2012) The role of lactoferrin binding protein B in mediating protection against human lactoferricin. Biochem Cell Biol 90(3):417-423. https://doi.org/10.1139/o11-074 PMID:22332888 
Morgenthau A, Pogoutse A, Adamiak P, Moraes TF, Schryvers AB (2013) Bacterial receptors for host transferrin and lactoferrin: molecular mechanisms and role in host-microbe interactions. Future Microbiol 8(12):1575-1585

Morgenthau A, Beddek A, Schryvers AB (2014) The negatively charged regions of lactoferrin binding protein $\mathrm{B}$, an adaptation against anti-microbial peptides. PLoS ONE 9(1):e86243. https://doi.org/10.1371/journal.pone.0086243

Noinaj N, Easley NC, Oke M, Mizuno N, Gumbart J, Boura E, Steere AN, Zak O, Aisen P, Tajkhorshid E, Evans RW, Gorringe AR, Mason AB, Steven AC, Buchanan SK (2012) Structural basis for iron piracy by pathogenic Neisseria. Nature 483:53-58. https://doi.org/10.1038/nature10823

Ogunnariwo JA, Schryvers AB (1990) Iron acquisition in Pasteurella haemolytica: expression and identification of a bovine-specific transferrin receptor. Infect Immun 58:2091-2097

Ogunnariwo JA, Schryvers AB (1992) Correlation between the ability of Haemophilus paragallinarum to acquire ovotransferrin-bound iron and the expression of ovotransferrin- specific receptors. Avian Dis 36:655-663

Ogunnariwo JA, Cheng CY, Ford JA, Schryvers AB (1990) Response of Haemophilus somnus to iron limitation: expression and identification of a bovine-specific transferrin receptor. Microb Pathog 9:397-406

Ogunnariwo JA, Alcantara J, Schryvers AB (1991) Evidence for non-siderophore-mediated acquisition of transferrin-bound iron by Pasteurella multocida. Microb Pathog 11:47-56

Ostan N, Yu R, Ng D, Chieh-Lin Lai C, Pogoutse A, Sarpe V, Hepburn M, Sheff J, Raval S, Schriemer DC, Moraes TF, Schryvers AB (2017) Lactoferrin binding protein B-A bifunctional bacterial receptor protein. PLoS Pathog 13(3):e1006244

Redfield RJ, Findlay WA, Bosse J, Kroll JS, Cameron AD, Nash JH (2006) Evolution of competence and DNA uptake specificity in the Pasteurellaceae. BMC Evol Biol 6:82. https://doi.org/10.1186/1471-2148-6-82

Rice P, Longden I, Bleasby A (2000) EMBOSS: the European molecular biology open software suite. Trends Genet 16(6):276-277

Rokbi B, Mignon M, Maitre-Wilmotte G, Lissolo L, Danve B, Caugant DA, Quentin-Millet M-J (1997) Evaluation of recombinant transferrin binding protein $\mathrm{B}$ variants from Neisseria meningitidis for their ability of induce cross reactive and bactericidal antibodies against a genetically diverse collection of serogroup B strains. Infect Immun 65(1):55-63

Roussel-Jazede V, Jongerius I, Bos MP, Tommassen J, van Ulsen P (2010) NalP-mediated proteolytic release of lactoferrin-binding protein $\mathrm{B}$ from the meningococcal cell surface. Infect Immun 78(7):3083-3089. https://doi.org/ 10.1128/iai.01193-09

Schaar V, Nordstrom T, Morgelin M, Riesbeck K (2011) Moraxella catarrhalis outer membrane vesicles carry beta-lactamase and promote survival of Streptococcus pneumoniae and Haemophilus influenzae by inactivating amoxicillin. Antimicrob Agents Chemother 55(8):3845-3853. https:// doi.org/10.1128/AAC.01772-10

Schryvers AB (1988) Characterization of the human transferrin and lactoferrin receptors in Haemophilus influenzae. Mol Microbiol 2:467-472
Schryvers AB (1990) A method for isolating and purifying transferrin and lactoferrin receptor proteins from bacteria and the preparation of vaccines containing the same. France Patent 052878700528787/EP B1

Schryvers AB, Gonzalez GC (1989) Comparison of the abilities of different protein sources of iron to enhance Neisseria meningitidis infection in mice. Infect Immun 57:2425-2429

Schryvers AB, Gonzalez GC (1990) Receptors for transferrin in pathogenic bacteria are specific for the host's protein. Can J Microbiol 36:145-147

Schryvers AB, Gray-Owen SD (1992) Iron acquisition in Haemophilus influenzae: receptors for human transferrin. J Infect Dis 165(1):S103-S104

Schryvers AB, Lee BC (1989) Comparative analysis of the transferrin and lactoferrin binding proteins in the family Neisseriaceae. Can J Microbiol 35(3):409-415

Schryvers AB, Morris LJ (1988a) Identification and characterization of the human lactoferrin-binding protein from Neisseria meningitidis. Infect Immun 56:1144-1149

Schryvers AB, Morris LJ (1988b) Identification and characterization of the transferrin receptor from Neisseria meningitidis. Mol Microbiol 2:281-288

Schryvers AB, Moraes TF, Gray-Owen SD (2014) Immunogenic compositions and vaccines derived from bacterial surface receptor proteins. PCT/CA2014/051146

Stearns JC, Davidson CJ, McKeon S, Whelan FJ, Fontes ME, Schryvers AB, Bowdish DM, Kellner JD, Surette MG (2015) Culture and molecular-based profiles show shifts in bacterial communities of the upper respiratory tract that occur with age. ISME J 9(5):1246-1259. https://doi.org/10. 1038/ismej.2014.250

Stojiljkovic I, Larson J, Hwa V, Anic S, So M (1996) HmbR outer membrane proteins of pathogenic Neisseriae: ironregulated, hemoglobin binding proteins with high degree of primary structure conservation. J Bacteriol 178:3341-3352

Tettelin H, Saunders NJ, Heidelberg J, Jeffries AC, Nelson KE, Eisen JA, Ketchum KA, Hood DW, Peden JF, Dodson RJ, Nelson WC, Gwinn ML, DeBoy R, Peterson JD, Hickey EK, Haft DH, Salzberg SL, White O, Fleischmann RD, Dougherty BA, Mason T, Ciecko A, Parksey DS, Blair E (2000) Complete genome sequence of Neisseria meningitidis serogroup B strain MC58. Science 287(5459):1809-1815

Turner S, Pryer KM, Miao VP, Palmer JD (1999) Investigating deep phylogenetic relationships among cyanobacteria and plastids by small subunit rRNA sequence analysis. J Eukaryot Microbiol 46(4):327-338

West D, Reddin K, Matheson M, Heath R, Funnell S, Hudson M, Robinson A, Gorringe A (2001) Recombinant Neisseria meningitidis transferrin binding protein A protects against experimental meningococcal infection. Infect Immun 69(3):1561-1567

Wilmotte A, Van der Auwera G, De Wachter R (1993) Structure of the $16 \mathrm{~S}$ ribosomal RNA of the thermophilic cyanobacterium Chlorogloeopsis HTF (Mastigocladus laminosus HTF) strain PCC7518, and phylogenetic analysis. FEBS Lett 317(1-2):96-100

Yu R-H, Schryvers AB (2002) Bacterial lactoferrin receptors: insights from characterizing the Moraxella bovis receptors. Biochem Cell Biol 80:81-90 